LA-UR- 0900321

Approved for public release; distribution is unlimited.
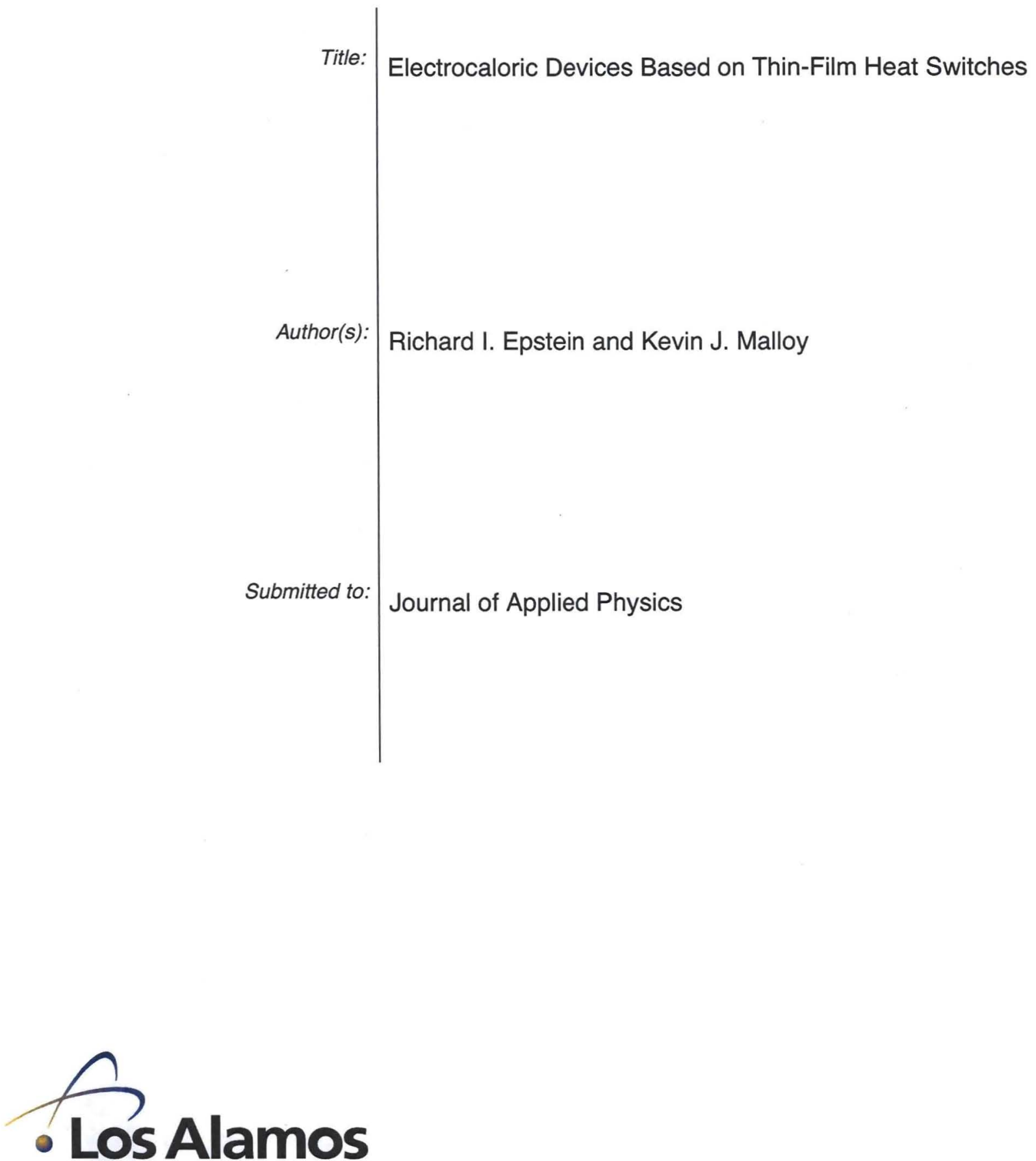

NATIONAL LABORATORY

Los Alamos National Laboratory, an affirmative action/equal opportunity employer, is operated by the University of California for the U.S. Department of Energy under contract W-7405-ENG-36. By acceptance of this article, the publisher recognizes that the U.S. Government retains a nonexclusive, royalty-free license to publish or reproduce the published form of this contribution, or to allow others to do so, for U.S. Government purposes. Los Alamos National Laboratory requests that the publisher identify this article as work performed under the auspices of the U.S. Department of Energy. Los Alamos National Laboratory strongly supports academic freedom and a researcher's right to publish; as an institution, however, the Laboratory does not endorse the viewpoint of a publication or guarantee its technical correctness. 


\title{
Electrocaloric devices based on thin-film heat switches
}

\author{
Richard I. Epstein ${ }^{1,2}$ and Kevin J. Malloy ${ }^{2}$ \\ ${ }^{1}$ Los Alamos National Laboratory, Los Alamos, NM 87545 \\ ${ }^{2}$ University of New Mexico, Albuquerque, NM 87131
}

\begin{abstract}
:
We describe a new approach to refrigeration and electrical generation that exploits the attractive properties of thin films of electrocaloric materials. Layers of electrocaloric material coupled with thin-film heat switches can work as either refrigerators or electrical generators, depending on the phasing of the applied voltages and heat switching. With heat switches based on thin layers of liquid crystals, the efficiency of these thin-film heat engines can be at least as high as that of current thermoelectric devices. Advanced heat switches would enable thin-film heat engines to outperform conventional vaporcompression devices.
\end{abstract}

\section{Introduction}

Recently, the demonstrations of giant electrocaloric effect (ECE) in thin films have underscored the potential for using ECE for efficient conversion of heat into electrical power or for refrigeration, air conditioning and heat pumping (Mischenko, et al. 2006; Neese et al. 2008). Nevertheless, no one has yet described how the ECE in thin films could be implemented in a practical device. Here we introduce the concept of thin-film heat switches and show how this makes efficient thin-film electrocaloric heat engines possible.

Applying an electric field to an electrocaloric material raises its temperature, and decreasing the field lowers its temperature. While there have been several reported schemes for exploiting bulk ECE for refrigeration, there have not been any detailed discussion of thin-film electrocaloric devices (Radebaugh et al. 1979; Olsen et al. 1985). Here we describe an approach to ECE heat engines based on stacks of thin films of electrocaloric materials interleaved with thin-film heat switches. This approach is scalable to support or exploit large temperature differences for refrigeration and power generation.

The thin-film heat switches needed for electrocaloric refrigerators and electrical generators must rapidly switch its thermal conductivity to regulate the heat flow between the electrocaloric films. In Section 2 we describe the general concept of thin-film electrocaloric heat engines. Section 3 presents one implementation of thin-film heat switches based on liquid crystal materials. Section 4 describes how stratified electrocaloric layers can be constructed so that high electric fields can be applied with relatively low voltages while maintaining thermal diffusion times that are compatible with the time scales of the heat switches. After that, we evaluate the thermodynamic performance of the thin films refrigerators (Section 5) and generators (Section 6). These calculations show that for sufficiently effective heat switches (with large conductivity contrasts between the open and closed states), thin-film heat engines would be more 
efficient than conventional vapor-compression devices. The final section compares thinfilm electrocaloric heat engines with thermoelectric and vapor compression devices.

In addition to their efficiency, thin-film heat engines have other significant practical advantages over other heat engines. They are lightweight devices that do not require any macroscopic motions of solid parts or fluids. These devices can be constructed from a large range of inexpensive and non-toxic materials. For example, some of the best electrocaloric materials are polymers. The availability of large sheets of efficient, inexpensive flexible thin-film heat engines would have a major impact on refrigeration, air conditioning, heat pumps and the reclamation of energy from waste heat.

\section{Overview}

To effectively use a stack of electrocaloric layers in a heat engine, the thermal connections between the layers has to be opened and closed appropriately as the layers are heated or cooled. A multilayer heat engine operates in a "bucket brigade" mode, rhythmically passing heat between adjacent electrocaloric layers (see Figure 1). Heat switches on both sides of each electrocaloric layer control the heat flows.

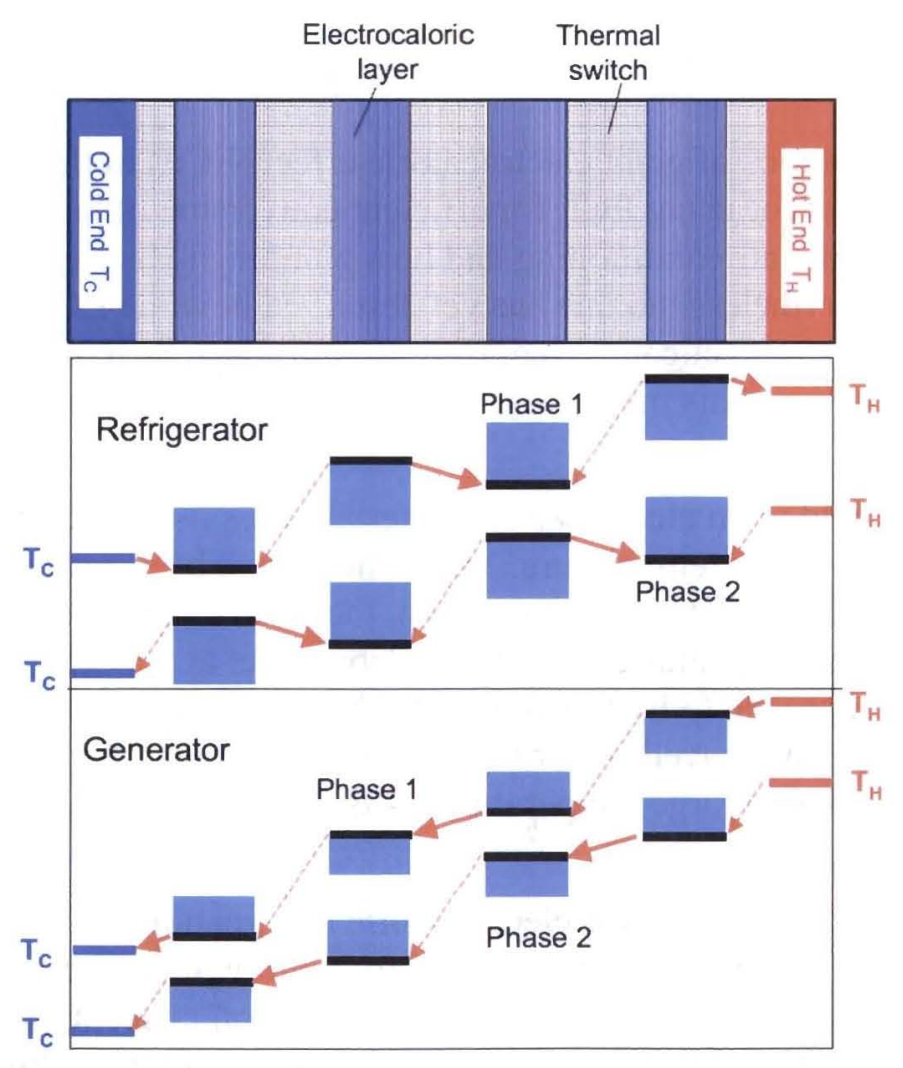

Figure 1. Operation of thin-film heat engines. The top panel shows the layers of electrocaloric material connected to each other and the hot and cold reservoir by heat switches. The middle panel shows the temperature profile of a refrigerator during the two isothermal phases. The bottom panel illustrates the temperature profiles for an electrical generator. The shaded regions in the bottom two panels indicate the temperature variations in each electrocaloric layer. The full arrows indicated the allowed heat flows and the dashed arrows show the suppressed heat flows. 
The top panel in Figure 1 is a schematic of a thin-film heat engine with four active layers. The electrocaloric layers are connected to the hot and cold ends of the device and to each other by heat switches. The middle panel shows the temperature profiles of the device during two phases of operation when it is functioning as a refrigerator, and the bottom panel shows the comparable temperature profiles for a generator. During Phase 1 for the refrigerator, the voltages across the electrocaloric layers are adjusted so that the first and third layers are cool relative to their average temperatures and the second and fourth are relatively warm. The heat switches are adjusted so that the net heat flows are to the right (from the cold reservoir to the first electrocaloric layer, from the second layer to the third, and from the fourth layer to the hot reservoir). During $t$ Phase 2 , the voltage are adjusted such that layers one and three are relatively warm and i layers two and four are cooler. The heat switches are reversed so that the heat continues to flow towards the right (from layer one to two and from layer three to four). The shaded regions show the temperature range through which the electrocaloric material shifts between Phases 1 and 2 . When the thin-film heat engine operates as an electrical generator, the heat flow is from the hot reservoir to the cold reservoir (to the left) and electrical power is extracted. The sequence of voltage and heat switch changes is similar to that of the refrigerator cycle. The important difference is that in the generator there is a net flow of heat into the electrocaloric material when it is hot and out of this material when it is cool, the reverse of what happens in the refrigerator.

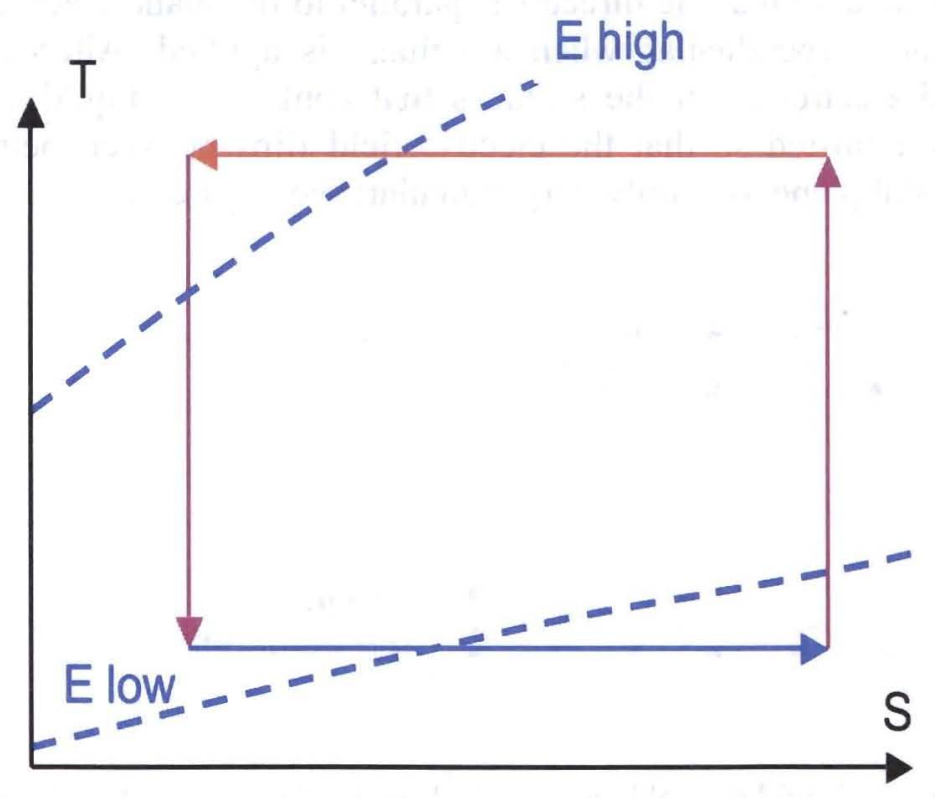

Figure 2. Thermodynamic cycle of a layer of electrocaloric material in a thin-film refrigerator. The direction of cycling reversed for an electrical generator.

The functioning of the thin-film refrigerator is illustrated in Figure 2, which shows the thermodynamic cycle of a single layer of electrocaloric material in the temperatureentropy plane. Two curves of constant electric field are shown to indicate how the applied electric field changes around the cycle. Each layer undergoes a Carnot cycle. A changing electric field drives the vertical, adiabatic legs of the cycle. A combination of heat flows and changing electric field maintains constant temperature in the horizontal isothermal legs. The efficiency of an actual thin-film heat engine is lower than the 
Carnot value because of entropy generation from heat flows though the heat switches and because of hysteresis in the electrocaloric material. The thermodynamic cycle for an electrical generator is the same, but with the direction of cycling reversed.

\section{Liquid crystal heat switches}

It has long been known that the thermal conductance of some liquid crystals is strongly anisotropic (Rondelez et al., 1978). For example, in some measured rod-like or calamitic liquid crystals the thermal conductivity are more than three times greater along the molecular director than perpendicular to it. Here we discuss how the thermal conductivity anisotropy of liquid crystals can be harnessed to make thin-film heat switches.

A heat switch would consist of a thin layer of liquid crystal and a mechanism for changing the orientation of liquid crystal's director from parallel to perpendicular to the plane of the film. When the director is mainly perpendicular to the film, the thermal conductivity across the film is enhanced to $\kappa_{\text {high }}$. When the director is mainly in the plane of the film and the thermal conductivity across the film is reduced to $\kappa_{\text {low }}$. The orientation of the liquid crystal directors can be controlled by electric fields applied across the liquid crystal. One possibility is that the surface of the material confining the liquid crystal can be textured so that the director is parallel to the plane when there is no applied electric field and perpendicular when a voltage is applied. Alternatively, by depositing interdigitated electrodes on the surfaces that confine the liquid crystal, the applied voltages can be adjusted so that the electric field flips between being mainly parallel to the liquid-crystal plane to mainly perpendicular; see Figure 3.

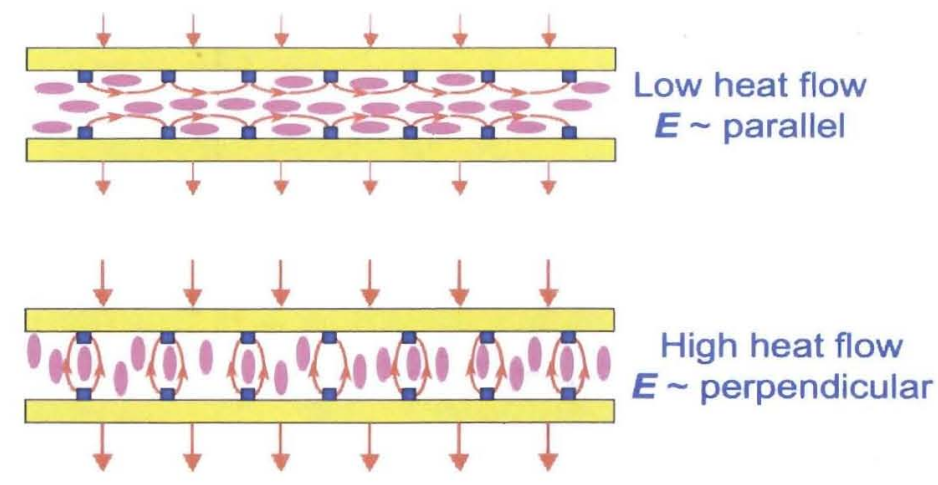

Figure 3. Edge view of a liquid crystal heat switch showing the interdigitated electrodes (rectangles) and the liquid crystal directors (indicated by the orientation of the ellipses). The electric field direction (curved arrows) and the liquid crystal directors can be switched between being predominately parallel to the film of liquid crystal (top panel) to mainly perpendicular to the film (bottom panel).

The time scale for the liquid crystal molecules to reorient decreases as the square of the applied electric field above a threshold value. For example, for the liquid crystals studied by Oh-E \& Kondo (1997), MLC-2011 and MLC-2806, the dynamic response times are $\sim 20 \mathrm{E}^{-2} \mathrm{~ms}$ and $\sim 40 \mathrm{E}^{-2} \mathrm{~ms}$, respectively, for high fields, where the electric field $E$ is in $\mathrm{MV} / \mathrm{m}$. Liquid crystal heat switches can be thinner than a micron, since unlike liquid 
crystal displays there is no need for large birefringence. Therefore, heat switches that use only a few volts can have switching time scales $t_{\text {switch }}$ of order a millisecond.

As is discussed below, the performances of the thin-film heat engines depends strongly on the ratio $K=\kappa_{\text {high }} / \kappa_{\text {low }}$ which is a measure of how well the heat switches operate. Larger values of $K$ allow one to build more efficient heat engines (see Figure 6, below). Various experiments indicate that heat flows rapidly along the length of the rod-like molecules but slowly between molecules (Urbach, et al., 1983; Pereira, et al., 2003). This interpretation is consistent with the observations that longer molecules of a given type tend to have larger $K$ values (Marinelli, et al., 1998). It may be possible to find liquid crystals with large the conductivity contrast $K$ by searching for long molecules which exhibit lower the thermal impedance between aligned molecules. Furthermore, combining carbon nanotubes with liquid crystals might enhance $K$ (Weiss, et al. 2006, Song et al. 2003). These nanotubes would essential act as exceptionally long molecules. When aligned with the liquid crystal director, they could greatly increase the heat transport anisotropy.

\section{Electrocaloric layers}

Above the Curie temperatures of electrocaloric materials, in the paraelectric regime, hysteretic losses can be quite small, and the materials can undergo rapid thermal cycling with little entropy production. Recently, researchers have found materials with large electrocaloric effects; Mischenko, et al., (2006) reported large electrocaloric effects in polycrystalline PZT and Neese et al. (2008) showed similar effects in ferroelectric polymers. In these studies the adiabatic temperature changes are of order $10 \mathrm{~K}$ for applied fields of $\sim 100 \mathrm{MV} \mathrm{m}^{-1}$. The need for such large electric fields constrains the design of an electrocaloric heat engine. Practical considerations of safety and convenience favor the use of relatively low voltages in heat engines. A heat engine can produce the needed high fields with low voltages if the electrodes on the electrocaloric material are separated by less than a micron.

Another consideration that has to be included in the design of the electrocaloric layers is the time scale for heat flow. Heat diffuses out of a layer of thickness $s$ in a time $t_{\text {diff }} \sim$ $s^{2} C / \kappa$, where, $C$ is the specific heat and $\kappa$ is the thermal conductivity. The response time of the heat switches has to be shorter than the heat diffusion time in order for switches to be able to control the heat flows. Efficiently functioning heat engines therefore require that $t_{\text {diff }}>t_{\text {switch }}>1 \mathrm{~ms}$. This condition sets a bound on the thickness of the electrocaloric layer.

$$
s>\left(\frac{t_{\text {switch }} \kappa}{C}\right)^{1 / 2} \sim 14\left(\frac{t_{\text {switch }}}{1 \mathrm{~ms}}\right)^{1 / 2} \mu \mathrm{m}
$$

where we used $\kappa / C \sim 500 \mathrm{~s} \mathrm{~cm}^{-2}$, which is within a factor of 2 of the values for the relevant polymers and for polycrystalline PZT. The constraint (1) applies to the entire thickness of an electrocaloric layer. If an electrocaloric layer is actually composed of many sub-micron films separated by electrodes the diffusion time can be made long 
relative to the response time of the heat switches and large electric fields can be produced with low voltages; see Figure 4.

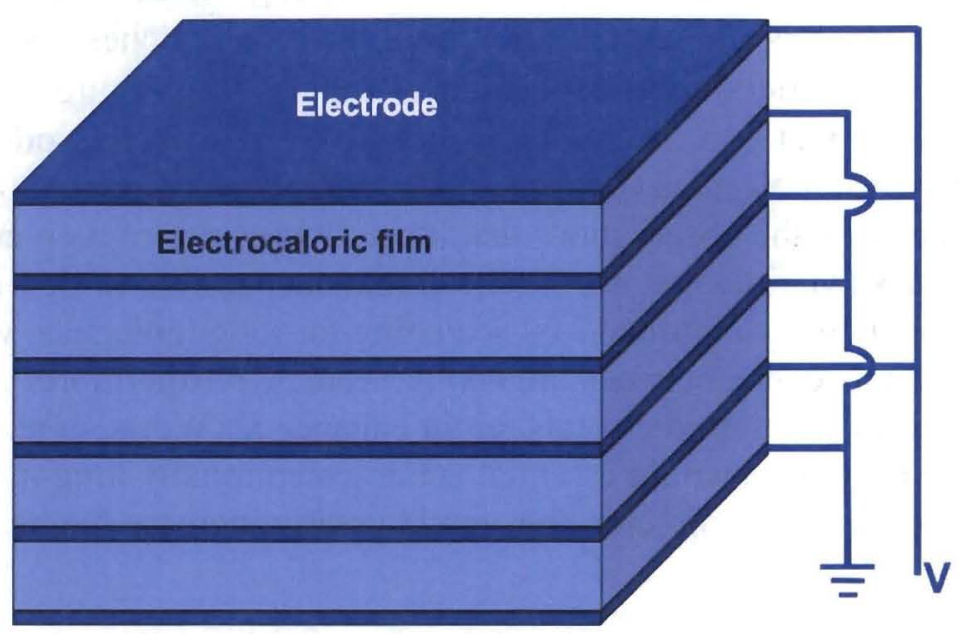

Figure 4. Electrocaloric layers consist of a stack of sub-micron films of electrocaloric material with electrodes on either side. This type of structure produces large electric fields in the electrocaloric material with low voltages.

\section{Efficiency and power density of thin-film refrigerators}

To evaluate the efficiency of a thin-film refrigerator we first consider a single-activelayer device as shown in Figure 5. A multilayer device, as shown in Figure 1, is essentially a concatenation of single-active-layer devices in which adjacent layers are half a cycle out of phase. In a multilayer device the "connection points" of the single-activelayer units are the cold and hot ends of the device and the midpoints between electrocaloric layers where the temperature does not change during a cycle. We assume that the switching time for the heat switches and the time needed to execute the adiabatic legs of the thermodynamic cycle (Figure 2) are short compared to the thermal diffusion time. These idealizations allow us to ignore heat flows during the adiabatic parts of the thermal cycle and to take the conductivity of the heat switches to be either $\kappa_{\text {high }}$ or $\kappa_{\text {low }}$.

Entropy generated by heat flows in the temperature gradients in the heat switches limits the efficiency of thin-film refrigerators (and generators). To calculate the device efficiency, we first tally the heat flows during the isothermal phases of the refrigeration cycle. We take the hot end of the refrigerator layer to be at temperature $T_{\mathrm{h}}=T(1+\Delta)$ and the cold end to be at $T_{\mathrm{c}}=T(1-\Delta)$; see Figure 5. During isothermal Phase 1, lowering the on the electrocaloric layer reduces its temperature to $T\left(1-\delta_{\mathrm{c}}\right)$. The heat switch to the cold end is set to high conductivity $\kappa_{\text {high }}$, and the switch to the hot end is set to low conductivity $\kappa_{\text {low. }}$. This configuration permits heat to preferentially flow from the cold end into the electrocaloric layer. During Phase 2, these operations are reversed. The temperature of the electrocaloric layer is raised to $T\left(1+\delta_{\mathrm{h}}\right)$ and the switches are adjusted so that heat flows to the hot end. The electrocaloric properties of the active material and 
the applied voltage layer determine the relative amplitude of the full temperature swing; we characterize this swing by $\delta=\left(\delta_{\mathrm{h}}+\delta_{\mathrm{c}}\right) / 2$.

During each full cycle, the device absorbs $Q_{c}$ in heat per unit area from the cold end and expels $Q_{\mathrm{h}}{ }^{\text {out }}$ heat at the hot end. Over a cycle the energy density that is supplied per cycle $W^{\text {in }}$ is given by

$$
W^{\text {in }}=Q_{\mathrm{h}}{ }^{\text {out }}-Q_{\mathrm{c}}{ }^{\text {in }} \text {. }
$$
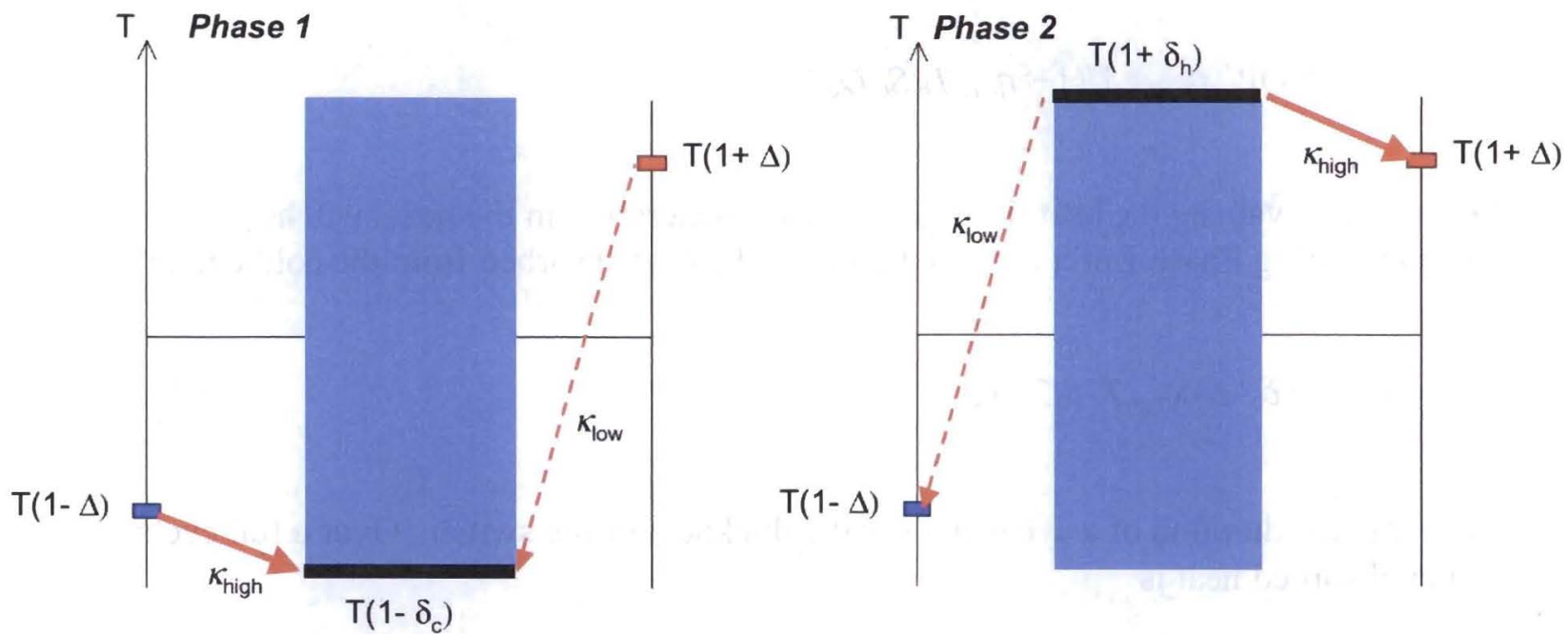

Figure 5. Heat flows in a section of a thin-film refrigerator. The solid arrows indicate the rapid heat flow through the heat switches with conductivity $\kappa_{\text {high }}$ and the dashed arrows represent the slow heat flow though the switches with conductivity $\kappa_{\text {low }}$.

The heat flowing down the temperature gradients in the heat switches generates entropy $S$ per cycle per unit area. The generated entropy plus that absorbed at the cold end is ejected at the hot end; i.e.,

$$
S+Q_{\mathrm{c}}^{\text {in }} / T_{\mathrm{c}}=Q_{\mathrm{h}}^{\text {out }} / T_{\mathrm{h}} \text {. }
$$

Using Equations (2) and (3), one can express the input work as

$$
W^{\text {in }}=T_{\mathrm{h}} \mathrm{S}+Q_{\mathrm{c}}^{\text {in }} / \eta_{\mathrm{CR}}
$$

where

$$
\eta_{\mathrm{CR}}=T_{\mathrm{c}} /\left(T_{\mathrm{h}}-T_{\mathrm{c}}\right)
$$


is the Carnot COP for a refrigerator. The coefficient of performance for the refrigerator is

$$
\mathrm{COP}=Q_{\mathrm{c}}^{\text {in }} / W^{\text {in }}=\eta_{\mathrm{CR}} /\left(1+\eta_{\mathrm{CR}} T_{\mathrm{h}} S / Q_{\mathrm{c}}{ }^{\text {in }}\right)
$$

The COP approaches the Carnot value $\eta_{\mathrm{CR}}$ when the generated entropy $S$ vanishes. The efficiency $\varepsilon_{R}$ of the refrigerator relative to Carnot is

$$
\varepsilon_{\mathrm{R}}=\mathrm{COP} / \eta_{\mathrm{CR}}=1 /\left(1+\eta_{\mathrm{CR}} T_{\mathrm{h}} S / Q_{\mathrm{c}}{ }^{\text {in }}\right) .
$$

We can now evaluate the heat flows and entropy generation in the heat switches. For example, during Phase 1 of a cycle in Figure 5, the heat absorbed from the cold end is

$$
Q_{\mathrm{cl}}=\left(\delta_{\mathrm{c}}-\Delta\right) \kappa_{\mathrm{high}} T \tau /(2 s),
$$

where $\tau$ is the duration of a cycle and $s$ is the thickness of the switch. Over a full cycle, the net absorbed heat is

$$
Q_{\mathrm{c}}^{\text {in }}=Q_{\mathrm{c} 1}+Q_{\mathrm{c} 2}=\left[\left(\delta_{\mathrm{c}}-\Delta\right) \kappa_{\text {high }}-\left(\delta_{\mathrm{h}}+\Delta\right) \kappa_{\mathrm{low}}\right] T \tau /(2 s),
$$

Where $Q_{\mathrm{c} 2}$ is the heat absorbed from the cold end during Phase 2 (a negative value). Similarly, the expelled heat at the hot end is

$$
Q_{\mathrm{h}}{ }^{\text {out }}=Q_{\mathrm{h} 1}+Q_{\mathrm{h} 2}=\left[\left(\delta_{\mathrm{h}}-\Delta\right) \kappa_{\mathrm{high}}-\left(\delta_{\mathrm{c}}+\Delta\right) \kappa_{\mathrm{low}}\right] T \tau /(2 s),
$$

Where $Q_{\mathrm{hk}}$ is the heat expelled during phase $k(=1,2)$. Inserting these expressions for the heat transport into Equation (2) for the work per cycle gives

$$
W^{\text {in }}=\left(\delta_{\mathrm{h}}-\delta_{\mathrm{c}}\right)\left(\kappa_{\text {high }}+\kappa_{\text {low }}\right) T \tau /(2 s) .
$$

We can see that the work expended per cycle is proportional to the offset between $\delta_{\mathrm{h}}$ and $\delta_{\mathrm{c}}$.

Entropy is generated by the heat flow through the temperature gradients in the switches. If the heat flow in a switch is $Q$ and the temperature at hot end is $T_{\mathrm{a}}$ and at the cold end is 
$T_{\mathrm{b}}$, then the entropy generated in the switch is $Q\left(T_{\mathrm{b}}^{-1}-T_{\mathrm{a}}^{-1}\right)$. During Phase 1 of the refrigerator cycle the generated entropy is

$$
S_{1}=Q_{1 \mathrm{c}}\left[\left(1-\delta_{\mathrm{c}}\right)^{-1}-(1-\Delta)^{-1}\right] / T-Q_{\mathrm{lh}}\left[\left(1-\delta_{\mathrm{c}}\right)^{-1}-(1+\Delta)^{-1}\right] / T,
$$

and during Phase 2 it is

$$
S_{2}=-Q_{2 \mathrm{c}}\left[(1-\Delta)^{-1}-\left(1+\delta_{\mathrm{h}}\right)^{-1}\right] / T+Q_{2 \mathrm{~h}}\left[(1+\Delta)^{-1}-\left(1+\delta_{\mathrm{h}}\right)^{-1}\right] / T
$$

The entropy generated per cycle is $S=S_{1}+S_{2}$.

To calculate the maximum relative efficiency $\varepsilon_{\mathrm{R}}$ we first equate the two expressions for the work (Equations 2 and 4 ) to solve for $\left(\delta_{\mathrm{h}}-\delta_{\mathrm{c}}\right)$ as a function of $K$ and $\Delta / \delta$. We then find the value of $\Delta / \delta$ that gives the maximum value of the $\varepsilon_{\mathrm{R}}$. These calculations can be simplified by keeping only the lowest order terms in relative temperature swing $\delta$; this is a good approximation because for the largest measured ECE the values of $\delta$ are $<0.02$ (Mischenko, et al. 2006; Neese et al. 2008). We find that the maximum efficiency relative to the Carnot value is

$$
\varepsilon_{R}=\left(\frac{\sqrt{\boldsymbol{K}}-1}{\sqrt{\boldsymbol{K}}+1}\right)^{2}
$$

This maximum efficiency is obtained when temperature span of the refrigerator and the temperature swing of the electrocaloric material are related by

$$
\frac{\Delta}{\delta}=\frac{\sqrt{\boldsymbol{R}}-1}{\sqrt{\boldsymbol{K}}+1}
$$

and when the difference between the mean temperature of the electrocaloric material and the mean temperature between the hot and cold reservoir is given by

$$
\frac{\delta_{h}-\delta_{c}}{\delta}=\frac{4 \sqrt{\boldsymbol{K}}}{\boldsymbol{K}+1} \delta
$$

When the thin-film refrigerator is at its maximum efficiency, the average heat from the cold end (the heat lift per unit area) is 


$$
F_{C}=\frac{T \kappa_{h i g h} \delta}{s} \frac{\sqrt{\not}-1}{\sqrt{\not \boldsymbol{A}}+\not \boldsymbol{A}} \equiv F_{C 0} \frac{\sqrt{\not{A}}-1}{\sqrt{\not{A}}+\not \boldsymbol{A}}
$$

The heat flux $F_{\mathrm{C}}$ increases linearly with $\delta$, since it is proportional to the temperature difference between layers. The temperature asymmetry $\delta_{\mathrm{h}}-\delta_{\mathrm{c}}$ (Equation 16 ) varies as $\delta^{2}$, and is generally quite small. This quadratic dependence is expected since the temperature asymmetry is proportional to input work, $\delta_{\mathrm{h}}-\delta_{\mathrm{c}} \sim W^{\text {in }}$ (Equation 11 ), $W^{\text {in }} \sim \eta_{\mathrm{CR}}{ }^{-1} F_{\mathrm{C}}$ and $\eta_{\mathrm{CR}}{ }^{-1} \sim F_{\mathrm{C}} \sim \delta$. Higher heat lifts than that given by Equation 17 are possible, if the thinfilm refrigerator is adjusted to operate at sub-optimal efficiency.

Figure 6 shows the relative efficiency $\varepsilon_{\mathrm{R}}$ and normalized heat lift $F_{\mathrm{C}} / F_{\mathrm{C} 0}$ versus the conductivity contrast $K$. The efficiency of the refrigerator approaches the Carnot value for very large $K$, while the optimal heat lift achieves its maximum near $K=6$.

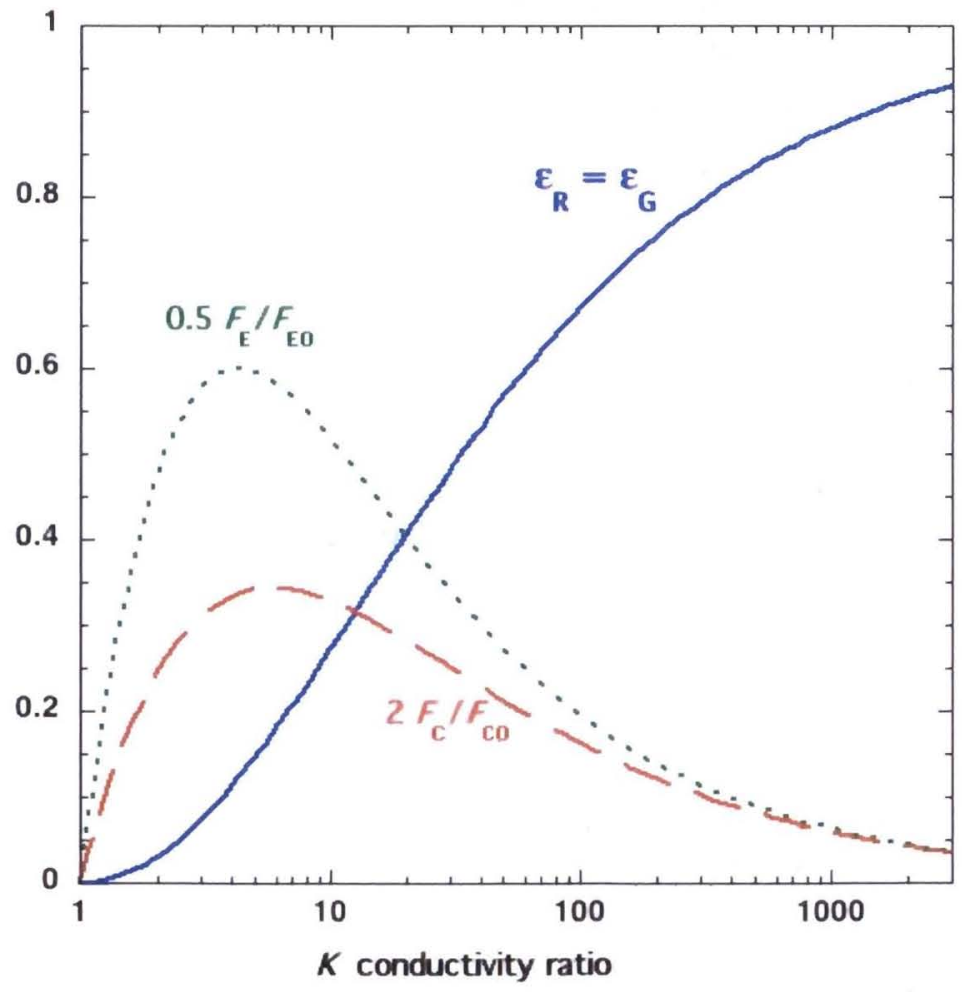

Figure 6. Properties of thin-film electrocaloric refrigerators and generators versus the conductivity contrast $K$ in the heat switches. The solid line gives the efficiency relative to the Carnot value for both refrigerators and generators. The dashed line shows the heat lift factor $F_{\mathrm{C}} / F_{\mathrm{C} 0}$, and the dotted line gives the electrical power generation parameter $F_{\mathrm{E}} / F_{\mathrm{E} 0}$. 
In a thin-film refrigerator composed of a stack of $N$ electrocaloric layers, the heat ejected into the hot reservoir $Q_{\mathrm{N}}$ at temperature $T_{\mathrm{N}}$ is related to the heat absorbed from the cold reservoir $Q_{0}$ at $T_{0}$ by

$$
Q_{N}=Q_{0}\left(1+\frac{1}{\varepsilon_{R} \eta_{C R}}\right)^{N}
$$

assuming $\varepsilon_{R} \eta_{C R}$ is the same in each layer. The overall coefficient of performance for the stack of $\mathrm{N}$ layers is

$$
\operatorname{COP}(N)=\frac{Q_{0}}{Q_{N}-Q_{0}} \equiv \varepsilon_{R}(N) \eta_{C R}(N),
$$

where the Carnot coefficient of performance for the stack is $\eta_{C R}(N)=T_{0} /\left(T_{N}-T_{0}\right)$, and $\varepsilon_{\mathrm{R}}(N)$ is the relative efficiency of the stack. In the usual case in which $T_{\mathrm{N}}-T_{0}<<T_{0}$, the above relations give $\varepsilon_{\mathrm{R}}(N) \sim \varepsilon_{\mathrm{R}}$; that is, the relative efficiency of the $N$-layer device is nearly the same as that of a single layer.

\section{Performance of thin-film generators}

Figure 7 illustrates the heat flows in a single-active-layer thin-film generator. This device produces electrical power as heat flows into and out of a central electrocaloric layer. The operation of this device is essential the reverse of the tin-film refrigerator. Again, the hot end is at $T_{\mathrm{h}}=T(1+\Delta)$ and the cold end is at $T_{\mathrm{c}}=T(1-\Delta)$. Now, $Q_{\mathrm{h}}$ is the heat absorbed at the hot end and $\mathrm{Q}_{\mathrm{c}}$ is the heat expelled at the cold end of the refrigerator and $W^{\text {out }}$ is the electrical work generated by the device. During isothermal Phase 1, increasing the voltage applied to the electrocaloric layer raises its temperature to $T\left(1+\delta_{\mathrm{c}}\right)$. The heat switch to the hot end is set to conductivity $\kappa_{\text {high }}$, and the switch to the cold end is set to $\kappa_{\text {low }}$. This configuration permits heat to preferentially flow from the hot end into the electrocaloric layer. During Phase 2, these operations are reversed.

Since the average heat flow is reversed for the generator and the refrigerator, the electrical energy extracted per unit area over a cycle is

$$
W^{\text {out }}=Q_{\mathrm{h}}{ }^{\text {in }}-Q_{\mathrm{c}}{ }^{\text {out }} \text {. }
$$

The expression for the entropy flows and production in the generator is now 

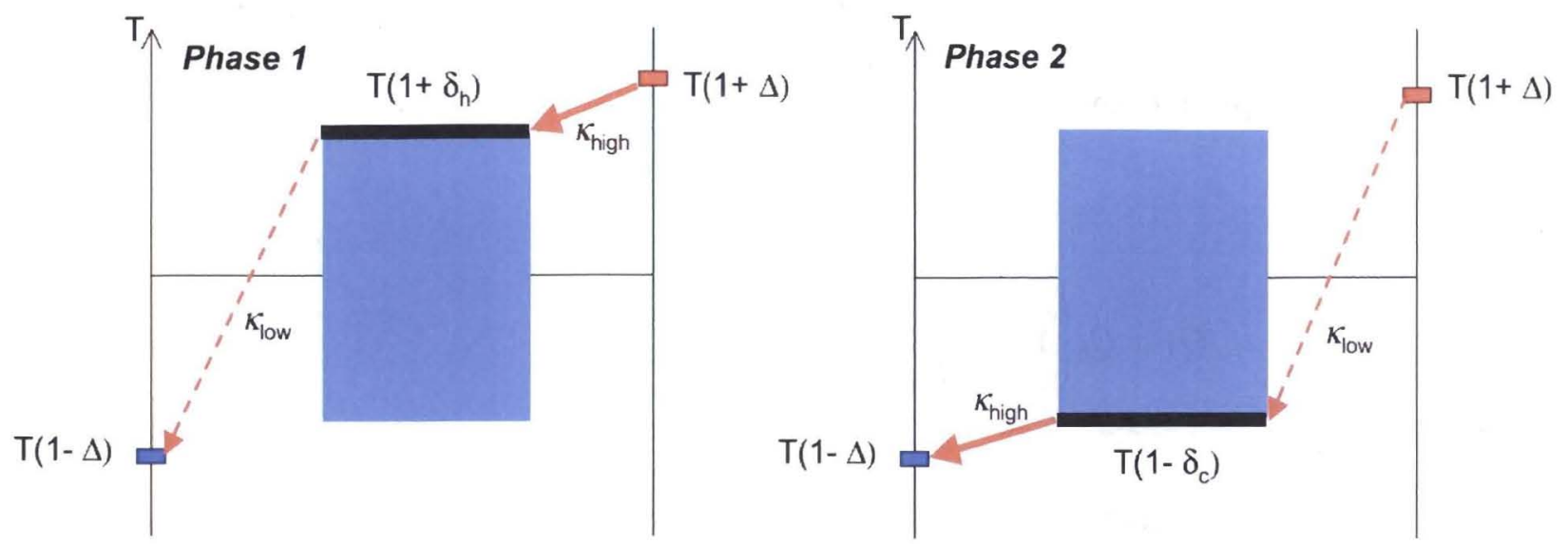

Figure 7. Heat flows in a section of a thin-film generator. As in Figure 5, the solid arrows indicate the rapid heat flows and the dashed arrows represent the slow heat flows.

$$
S+Q_{\mathrm{h}}{ }^{\text {in }} / T_{\mathrm{h}}=Q_{\mathrm{c}}{ }^{\text {out }} / T_{\mathrm{c}} .
$$

Using Equations (20) and (21), the work and the efficiency of the generator $\eta_{\mathrm{G}}$ can be expressed as

$$
\begin{aligned}
& W^{\text {out }}=\eta_{\mathrm{CG}} Q_{\mathrm{h}}{ }^{\text {in }}-T_{\mathrm{c}} S \\
& \eta_{\mathrm{G}}=W^{\text {out }} / Q_{\mathrm{h}}=\eta_{\mathrm{CG}}-T_{\mathrm{c}} S / Q_{\mathrm{h}}{ }^{\text {in }}
\end{aligned}
$$

where

$$
\eta_{\mathrm{CG}}=\left(T_{\mathrm{h}}-T_{\mathrm{c}}\right) / T_{\mathrm{h}}
$$

is the Carnot efficiency for generators. The efficiency $\varepsilon_{\mathrm{G}}$ of the generator relative to Carnot is

$$
\varepsilon_{\mathrm{G}}=\eta_{\mathrm{G}} / \eta_{\mathrm{CG}}=1-T_{\mathrm{c}} S /\left(\eta_{\mathrm{CG}} Q_{\mathrm{h}}{ }^{\text {in }}\right)
$$

The expressions for heat flows and entropy generation for the generator are similar to those for the thin-film refrigerator (Equations 8-13). To calculate the maximum relative efficiency $\varepsilon_{\mathrm{G}}$ we equate the two expressions for the work (Equations 20 and 22) and obtain the offset $\left(\delta_{\mathrm{h}}-\delta_{\mathrm{c}}\right)$ in terms of $K$ and $\Delta / \delta$. We then find the value of $\Delta / \delta$ that gives the maximum value of $\varepsilon_{\mathrm{G}}$. Keeping only the lowest order terms in $\delta$, we find 


$$
\varepsilon_{G}=\left(\frac{\sqrt{K}-1}{\sqrt{K}+1}\right)^{2} .
$$

This expression is the same as the one we found for the maximum relative efficiency $\varepsilon_{R}$ for the thin-film refrigerator. For generator tuned to its optimal efficiency the device parameters are

$$
\frac{\Delta}{\delta}=\frac{\sqrt{K}+1}{\sqrt{K}-1},
$$

which is the reciprocal of the relation for the refrigerator, and

$$
\frac{\delta_{h}-\delta_{c}}{\delta}=-\frac{4 \sqrt{K}}{K+1} \delta
$$

this offset is the same magnitude as in the refrigerator (Equation 16), though of the opposite sense ( $\delta_{\mathrm{c}}$ is now larger than $\delta_{\mathrm{h}}$ ). When the thin-film generator runs at maximum efficiency it generates power at average rate per unit area of

$$
F_{E}=\frac{T \kappa_{\text {high }} \delta^{2}}{s} \frac{4(K-1)}{\sqrt{K}(\boldsymbol{K}+1)} \equiv F_{E 0} \frac{4(K-1)}{\sqrt{\boldsymbol{K}}(\boldsymbol{K}+1)} .
$$

Figure 6 shows $\varepsilon_{\mathrm{G}}$ and $F_{\mathrm{E}} / F_{\mathrm{E} 0}$ versus the conductivity contrast $K$.

For a thin-film generator is composed of a stack of $N$ electrocaloric layers, the heat ejected into the cold reservoir $Q_{\mathrm{N}}$ at temperature $T_{\mathrm{N}}$ is related to the heat absorbed from the hot reservoir $Q_{0}$ at $T_{0}$ by

$$
Q_{N}=Q_{0}\left(1-\varepsilon_{G} \eta_{C G}\right)^{N},
$$

taking $\varepsilon_{G} \eta_{C G}$ to be the same in each layer. The overall coefficient of performance for the stack of $\mathrm{N}$ layers is

$$
\eta_{G}(N)=\varepsilon_{G}(N) \eta_{C G}(N)=\frac{Q_{0}-Q_{N}}{Q_{0}},
$$

where the Carnot efficiency for the stack is $\eta_{C G}(N)=\left(T_{0}-T_{N}\right) / T_{0}$, and $\varepsilon_{\mathrm{G}}(N)$ is the relative efficiency of the stack. For $T_{\mathrm{N}}-T_{0}<<T_{0}$, the above relations give $\varepsilon_{\mathrm{G}}(N) \sim \varepsilon_{\mathrm{G}}$; that is, the relative efficiency of the $N$-layer device is nearly that of a single layer. 


\section{Discussion:}

Thin-film heat engines provide an approach for exploiting the electrocaloric effect for refrigeration and energy generation. The efficiency of these devices can be high if heat switches with large thermal conductivity contrasts $K$ are used. For even moderate values of $K$ the efficiency of thin-film heat engines can compare favorably with current thermoelectric devices. For small temperature differences the efficiency of ideal thermoelectric refrigerators or generators is

$$
\varepsilon_{T E}=\frac{\eta_{T E}}{\eta_{C}}=\frac{(1+Z T)^{1 / 2}-1}{(1+Z T)^{1 / 2}+1}
$$

where $Z T$ is the thermoelectric figure of merit. We can define an equivalent thin-film figure of merit $(Z T)_{\mathrm{TF}}$ by equating $\varepsilon_{\mathrm{TE}}$ and $\varepsilon_{\mathrm{G}}=\varepsilon_{\mathrm{R}}$. This calculation gives

$$
(Z T)_{T K} \doteq \frac{(K-1)^{2}}{4 K}
$$

This means, for example, that an ideal thin-film heat engines that have heat switches with conductivity contrasts of $K=20$ operate with the same efficiency as thermoelectric devices with figure of merit $Z T=4.5$. If higher contrast heat switches are used, thin-film heat engines could become more efficient than standard vapor-compression devices. Conductivity contrasts above $K=100$ correspond to relative efficiencies exceeding $65 \%$ of the Carnot value, considerably higher than most commercial vapor compression devices.

The cooling power density and the electrical power generation density vary strongly with the conductivity of the closed heat switch $\kappa_{\text {high }}$, the temperature swing $\delta$ of the electrocaloric layers, and the thickness of the heat switch $s$. For plausible fiducial values of $\kappa_{\text {high }} \sim 0.1 \mathrm{~W} \mathrm{~cm}, \delta \sim 0.01$ and $s=10 \mu \mathrm{m}$, the cooling power density is

$$
F_{C} \simeq 300\left(\frac{\kappa_{h i g h}}{0.1 \mathrm{~W} \mathrm{~cm}}\right)\left(\frac{\delta}{0.01}\right)\left(\frac{s}{10 \mu \mathrm{m}}\right)^{-1} \frac{F_{C}}{F_{C 0}} \mathrm{~W} \mathrm{~cm}^{-2}
$$

and the power generation density is

$$
F_{E} \simeq 3\left(\frac{\kappa_{\text {high }}}{0.1 \mathrm{~W} \mathrm{~cm}}\right)\left(\frac{\delta}{0.01}\right)^{2}\left(\frac{s}{10 \mu \mathrm{m}}\right)^{-1} \frac{F_{E}}{F_{E 0}} \mathrm{~W} \mathrm{~cm}^{-2},
$$

where $F_{\mathrm{C}} / F_{\mathrm{C} 0}$ and $F_{\mathrm{E}} / F_{\mathrm{E} 0}$ are shown in Figure 6 . These power densities are adequate for most practical cooling and energy reclamation applications. 
The development of thin-film heat switches with large contrasts $K$ between the open and closed states would enable thin-film electrocaloric heat engines that are more efficient than current commercial devices. Additionally, these devices would be attractive because of their low mass, lack of moving parts, and that they can be built from materials that are benign to the environment.

\section{References:}

Marinelli, M., Mercuri, F., Zammit, U. \& Scudieri, F., "Thermal conductivity and thermal diffusivity of the cyanobiphenyl (nCB) homologous series."Phys. Rev. E, 58, $5860,1998$.

Mischenko, A. S., Zhang, Q., Scott, J. F., Whatmore, R. W. \& Mathur, N. D., Science, 311, 1270, 2006.

Neese, B., Chu, B., Lu, S.-G., Wang, Y., Furman, E. \& Zhang, Q. M., "Large electrocaloric effect in ferroelectric polymers near room temperature", Science, 321, $821,2008$.

Olsen, R. B., Bruno, D. A. \& Briscoe, J. M., "Pyroelectric conversion cycles", J. Appl. Phys., 58, 4709, 1985.

Oh-E, M \& Kondo, K, "The in-plane switching of homogeneously aligned nematic liquid crystals", Liquid Crystals, 22, 379-390, 1997.

Pereira, J. R. D., et al. "Thermal diffusivity anisotropy in calamitic-nematic lyotropic liquid crystal”, Rev. Sci. Instr, 74, 822-824, 2003.

Radebaugh, R., Lawless, W. N., Siegwarth, J. D. \& Morrow, A. J., Cryogenics, 19, 187, 1979.

Rondelez, F., Urbach, W., \& Hervet, H., "Origin of Thermal Conductivity Anisotropy in Liquid Crystalline Phases”, Phys. Rev. Lett. 41, 1058, 1978.

Song, W.H., Kinloch, I.A, \& Windle, A.H., "Nematic liquid crystallinity of multiwall carbon nanotubes" Science, 302, 1363-1363, 2003.

Urbach, W. et al., "Thermal diffusivity in mesophases: A systematic study in 4-4'-di-(nalkoxy) azoxy benzenes" J. Chem. Phys, 78, 5113-5124, 1983.

Weiss, V., Thiruvengadathan, R. \& Regev, O., "Preparation and Characterization of a Carbon Nanotube-Lyotropic Liquid Crystal Composite", Langmuir, 22, 854-856, 2006. 